\title{
Herlyn-Werner-Wunderlich Syndrome: A Rare Müllerian Anomaly
}

\author{
Santosh B Jaybhaye
}

\begin{abstract}
A syndrome complex consisting of didelphys uteruses, obstructed hemivagina and ipsilateral renal agenesis is one of the most complex and rare congenital anomalies of the female urogenital system. In most of the cases patients remain asymptomatic till puberty only to present with dysmenorrhea, pelvic pains, and intermenstrual bleeding or abdominal lumps in the period following menarche. We present one of the atypical delayed presentations of this syndrome at 26 years of age with dysmenorrhea and irregular vaginal bleeding. Appropriate combination of imaging, clinical examination, and hysteroscopic evaluation confirmed the diagnosis followed by appropriate surgical correction to relieve the patient of her complaints.
\end{abstract}

Keywords: Müllerian duct anomaly, OHVIRA syndrome, Renal agenesis, Uterine didelphys.

Journal of South Asian Federation of Menopause Societies (2019): 10.5005/jp-journals-10032-1167

\section{Case Description}

A 26-year-old unmarried female presented to the outpatient department with complaints of severe dysmenorrhea, pain in abdomen for 6-7 years along with complaints of intermenstrual bleeding not responding to multiple attempts of hormonal treatment attempted by the primary general practitioner. No history of any major medical or surgical illness was reported in past.

\section{Menstrual History}

Patient gives a history of heavy menstrual flow for 7 days every 30-day cycle associated with dysmenorrhea followed by intermenstrual bleeding of altered brownish color almost daily throughout the month.

USG features were suggestive of bicornuate uterus with minimal hematometra on right side (Fig. 1) with absent right kidney (Fig. 2).

Patient had a history of hysterolaparoscopy done to evaluate the problem which confirmed the presence of bicornuate uterus. There was no evidence of endometriosis on laparoscopy.

Right uterine horn could not be negotiated during the last hysteroscopic attempt but there was discharge of hematometra collection from a small fistulous opening in the vaginal wall.

On clinical examination, general clinical examination was unremarkable without any significant positive finding. Per speculum examination revealed a bulge in right anterolateral vaginal wall, purulent discharge noted from a fistulous opening in the vaginal wall bulge on pressing (Fig. 3). Cervix more on left side is normal looking. On this much clinical evaluation, clinical diagnosis of Herlyn-Werner-Wunderlich Syndrome was made and patient was posted for hysteroscopic confirmation of diagnosis followed by surgical correction.

Intraoperatively on vaginoscopic examination the findings of perspeculum examinations were confirmed. Left-sided cervix could be negotiated with hysteroscope and a banana shaped right hemiuterine cavity with centrally placed ostium was noted. The hysteroscope was withdrawn and pushed through a small fistulous opening on the bulging right anterolateral vaginal wall. The hysteroscope entered into a blind vaginal pouch with pus
Department of Gynaecological Endoscopy, Om Sai Hospital, Panvel, Maharashtra, India

Corresponding Author: Santosh B Jaybhaye, Department of Gynaecological Endoscopy, Om Sai Hospital, Panvel, Maharashtra, India, Phone: +91 9819294537, e-mail: santojoy@yahoo.com

How to cite this article: Jaybhaye SB. Herlyn-Werner-Wunderlich Syndrome: A Rare Müllerian Anomaly. J South Asian Feder Menopause Soc 2019;7(1):34-36.

Source of support: Nil

Conflict of interest: None

collection. Right-sided cervix was located in the vaginal pouch and on negotiating the cervical right hemiuterine cavity could be visualized hysteroscopically. Uterine cavity was also draining purulent material. After this evaluation to confirm the suspected diagnosis of Herlyn-Werner-Wunderlich Syndrome the oblique obstructing vaginal septum was excised (Fig. 4) and the excised edges marsupulized with the vaginal wall to relieve the obstruction as well as to prevent reocclusion of outflow track. Final picture after

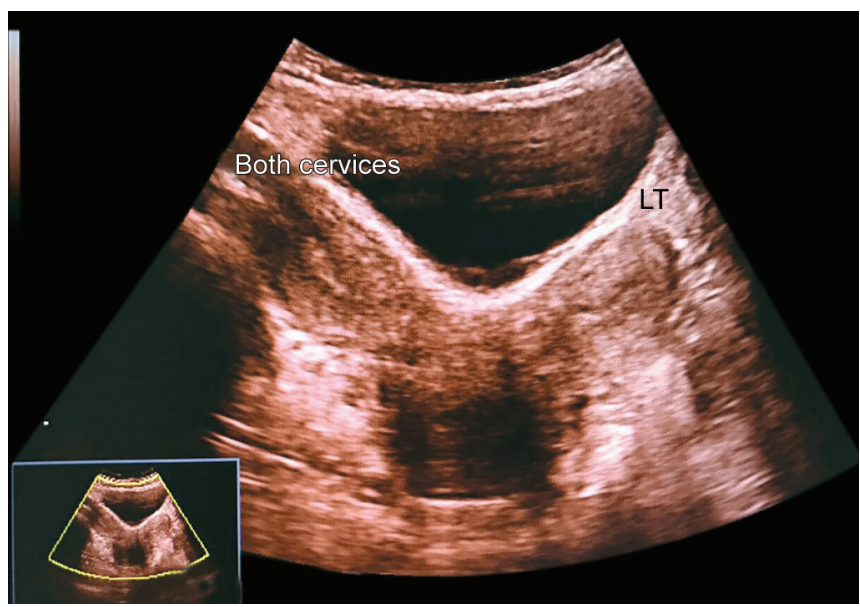

Fig. 1: Sonographic image showing bicornuate uterus

() The Author(s). 2019 Open Access This article is distributed under the terms of the Creative Commons Attribution 4.0 International License (https://creativecommons. org/licenses/by-nc/4.0/), which permits unrestricted use, distribution, and non-commercial reproduction in any medium, provided you give appropriate credit to the original author(s) and the source, provide a link to the Creative Commons license, and indicate if changes were made. The Creative Commons Public Domain Dedication waiver (http://creativecommons.org/publicdomain/zero/1.0/) applies to the data made available in this article, unless otherwise stated. 


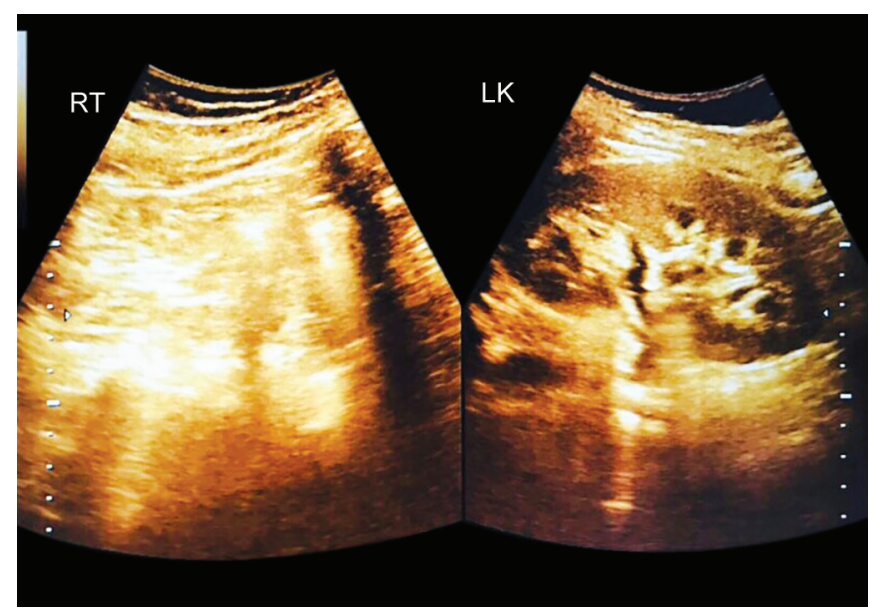

Fig. 2: Sonographic image suggesting right renal agenesis

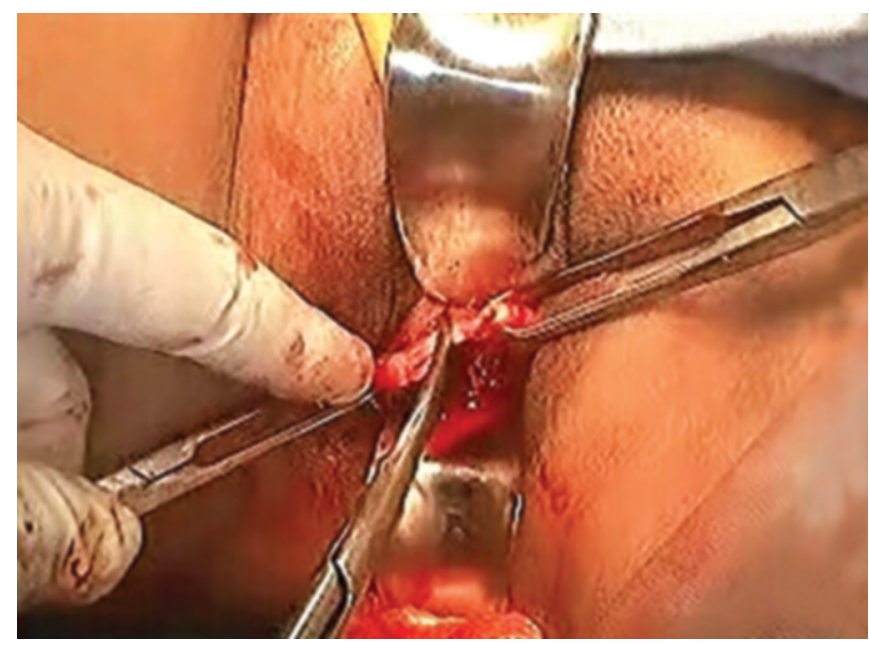

Fig. 4: Actual intraoperative photograph showing septum excision in progress

completion of excision as well as marsupialization resulted in the formation of a single vagina with two cervices sharing common medial wall (Fig. 5). Postoperative course was uneventful. The patient reported complete cure of her symptoms on 2 months follow-up postoperative visit.

\section{Discussion}

The Herlyn-Werner-Wunderlich Syndrome also known as OHVIRA (Obstructed Hemivagina, Ipsilateral Renal Agenesis) syndrome is characteristically found to be associated with uterine didelphys but occasionally it is also seen in combination with a septate uterus and is included in class III of American Fertility Society (AFS) Classification of Müllerian Anomalies. Incidence of this condition in various case series is $0.1-3.8 \% .^{1}$ Ipsilateral renal agenesis on the side of obstructed hemivagina is the most commonly associated urologic anomaly in OHVIRA syndrome, although few other malformations, like renal duplication and multicystic dysplastic kidney, have also been reported in some papers. ${ }^{2,3}$ Exact etiopathogenesis of this complex mullerian anomaly is eluding the clinicians since long time. Classical theory of female urogenital development was found to be inadequate in explaining the development of such complex mullerian anomalies. Multiple possibilities are proposed by many people. Acien proposed that while uterus and cervix were derived from fused-paired paramesonephric ducts (2nd part) and

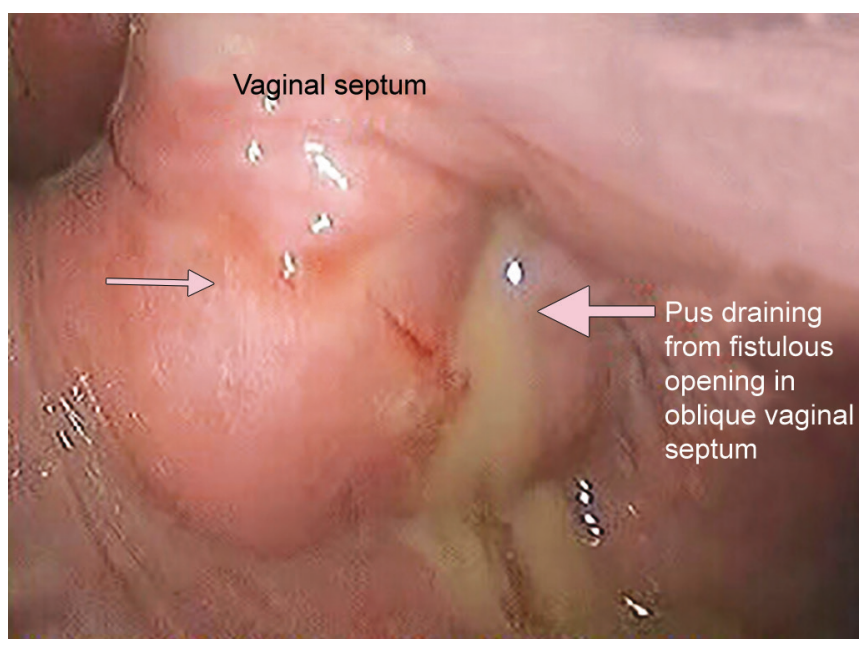

Fig. 3: Actual intraoperative photograph showing pus discharge from blind vaginal pouch

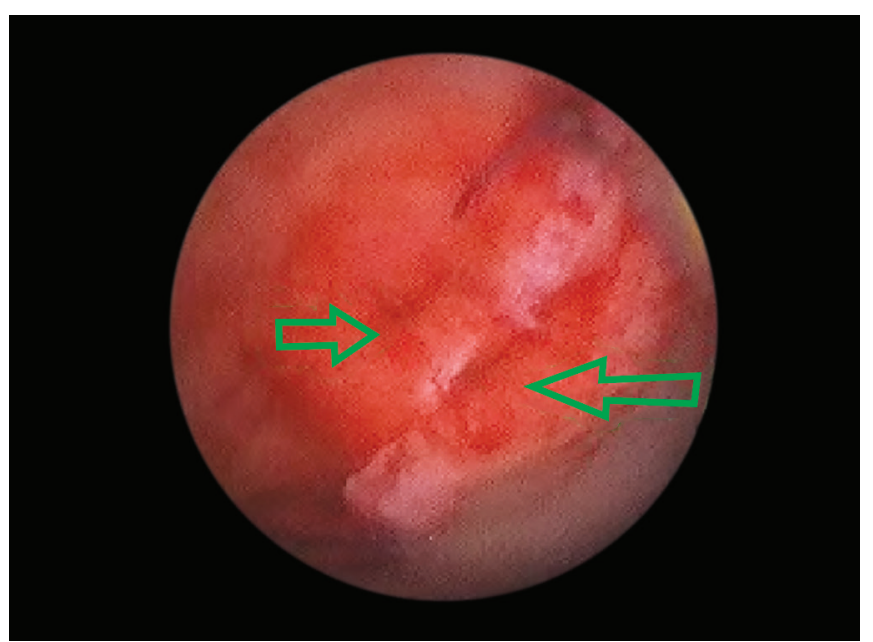

Fig. 5: Final intraoperative look showing a single vagina with two cervices sharing common medial wall

divergent distal paramesonephric ducts (3rd part), the vagina development is completely mesonephric in origin ${ }^{4}$ although its lining reveals mullerian cells derived from mullerian tubercle. Hence, paramesonephric ducts do not have any contribution to the embryonic development of the vagina although mullerian tubercle does, such that the vagina is lined by cells from mullerian tubercle. This theory has been further confirmed by data published by Sanchez in his experiments with female rats using immunohistochemistry techniques.

An early failure of metanephric diverticulum to develop (around 5 weeks) from mesonephric duct results in agenesis of ureteric bud, which leads to agenesis of ipsilateral ureter and kidney. Anatomical defect is created because of failed lateral fusion of mullerian ducts (didelphys/septate uterus and double vagina) in combination with complete or partial failure of longitudinal fusion between the mullerian duct and the urogenital sinus (hemivaginal septum) on the side of the renal agenesis. In a reported case series of 27 cases, the age at diagnosis varied from 10 to 29 years, with a median age of 14 years. ${ }^{3}$ Earliest reported case in the literature is a one-day-old neonate at birth where the diagnosis was suspected because of the antenatal scan showing absent right kidney and at birth, there was a prolapse noted over the infant's vaginal introitus measuring 
$3 \mathrm{~cm} \times 5 \mathrm{~cm}$ and was covered in a mucoid, bloody exudate. Detailed imaging confirmed the diagnosis. ${ }^{7}$

Defective development of both mullerian and Wolffian ducts has been proposed as one of the theories in development of this malformation. It is proposed that the Wolffian duct not only helps in creation of urinary collection systems but also a responsible factor for fusion of mullerian ducts. And hence developmental anomaly in the distal portion of the Wolffian duct results in ipsilateral renal agenesis as well as imperforate hemivagina. ${ }^{5}$

The patient usually presents after menarche with nonspecific symptoms of recurrent pelvic pain or dysmenorrhea from progressive distension of the obstructed hemivagina. Early and accurate diagnosis of this clinical entity is crucial because prompt surgical correction can relieve symptoms and prevent further complications related to chronic obstructive hematocolpos (such as endometriosis and pelvic adhesions), and maintain reproductive capacity. Delays in diagnosis were usually attributed to a lack of knowledge of this clinical entity, regular menstruations in the context of an incomplete vaginal outlet obstruction, and slow extension of hematocolpos. Surgical correction not only relieves pain with immediate effect but also reduces chances of pelvic endometriosis due to retrograde menstruation. In postoperative phase patients are capable of having a normal sexual life. In the earlier days hemihysterectomy was the treatment chosen for many patients. More and more addition of knowledge about this condition in the past few years has changed the treatment plans from hemihysterectomy to a simple excision of oblique complete vaginal septum relieving the obstruction.

\section{Conclusion}

OHVIRA syndrome is a rare congenital urogenital anomaly with a wide range of clinical presentation of simple surgical management. Imaging, particularly an MRI, plays a major role in diagnosis, which is often missed clinically. ${ }^{6}$ The complex mullerian anomalies have been difficult to diagnose correctly without help of imaging. Appropriate surgery is a single stage procedure to either excise or completely divide the obstructing septum. Early and correct diagnosis is required to relieve the symptoms and prevent complications caused by retrograde menstruation which may result in endometriosis and, also, preserve sexual functions and fertility.

\section{References}

1. Resetkova N, Christianson M, et al. Uterine Didelphys With Obstructed Hemivagina And Ipsilateral Renal Agenesis With Hydronephrosis. Fertil Steril 2012;97:30-31. DOI: 10.1016/j.fertnstert.2012.01.076.

2. Li S, Qayyum A, et al. Association Of Renal Agenesis And Mullerian Duct Anomalies. J Comput Assist Tomogr 2000;24:829-834. DOI: 10.1097/00004728-200011000-00001.

3. Vercellini P, Daguati R, et al. Asymmetric Lateral Distribution Of Obstructed Hemivagina And Renal Agenesis In Women With Uterus Didelphys: Institutional Case Series And A Systematic Literature Review. Fertil Steril 2007;87:719-724. DOI: 10.1016/ j.fertnstert.2007.01.173.

4. Acién P. Embryological Observations On The Female Genital Tract. Hum Reprod 1992;7:437-445. DOI: 10.1093/oxfordjournals.humrep. a137666.

5. Orazi C, Lucchetti MC, et al. Herlyn-Werner-Wunderlich Syndrome: Uterus Didelphys, Blind Hemivagina And Ipsilateral Renal Agenesis. Sonographic And Mr Findings In 11 Cases. Pediatr Radiol 2007;37: 657-665. DOI: 10.1007/s00247-007-0497-y.

6. Marten K, Vosshenrich R, et al. MRI In The Evaluation Of Mullerian Anomalies. Clin Imaging 2003;27:346Y350. DOI: 10.1016/S08997071(02)00587-9.

7. Wu T-H, Wu T-T, et al. Herlyn-Werner-Wunderlich Syndrome Consisting of Uterine Didelphys, Obstructed Hemivagina and Ipsilateral Renal Agenesis in a New-born. Pediatr Neonatol February 2012;53(1):68-71. DOI: 10.1016/j.pedneo.2011.11.014. 